\title{
Transanal minimally invasive surgery: from transanal minimally invasive surgery to pure natural orifice transluminal endoscopic surgery
}

\author{
Won Jun Jeong ${ }^{1,2}$, Byung Jo Choi ${ }^{1,2}$, Sang Chul Lee ${ }^{1,2}$ \\ 'Department of Surgery, Daejeon St. Mary's Hospital, Daejeon 94349, Republic of Korea. \\ ${ }^{2}$ Department of Surgery, College of Medicine, the Catholic University of Korea, Seoul 06591, Republic of Korea.
}

\begin{abstract}
Correspondence to: Prof. Sang Chul Lee, Department of Surgery, Daejeon St. Mary's Hospital, College of Medicine, The Catholic University of Korea, 520-2 Daeheung-dong, Jung-gu, Daejeon 301-723, Republic of Korea.

E-mail: zambo9@catholic.ac.kr; zambo9@hanmail.net
\end{abstract}

How to cite this article: Jeong WJ, Choi BJ, Lee SC. Transanal minimally invasive surgery: from transanal minimally invasive surgery to pure natural orifice transluminal endoscopic surgery. Mini-invasive Surg 2019;3:38.

http://dx.doi.org/10.20517/2574-1225.2019.42

Received: 15 Oct 2019 Accepted: 3 Dec 2019 Published: 12 Dec 2019

Science Editor: Xavier Serra-Aracil Copy Editor: Jing-Wen Zhang Production Editor: Tian Zhang

\section{ANATOMICAL CHARACTERISTICS OF RECTUM}

The rectum, the last stage of the digestive tract, inevitably crosses the pelvic cavity to the anus. The pelvic cavity is a tunnel-shaped space consisting of pelvic bones and is compactly filled with soft tissues including urogenital organs, blood vessels, nerves, and lymph nodes as well as the rectum. Rectal surgery performed in this narrow and visually adverse environment is difficult, regardless of surgical modalities in terms of firm procedures, securing margins, and nerve and blood vessel preservation. This is particularly acute in the distal rectum, because the rectum travels forward and close to the prostate and seminal vesicles in men and the vaginal wall in women.

\section{HISTORY OF RECTAL SURGERY}

The first description of rectal cancer was given by Joannes Baptista (1682-1771), and the first operation was performed by Jacques Lisfranc in 1826 in a transanal approach. The first colostomy to relieve large bowel obstruction was performed by Amussat in 1839 and the first transsacral approach to rectal cancer by Kraske in 1885. The combining of transabdominal and transperineal approaches to rectal cancer was already published by Czerny and Mayo in 1884 and 1904, respectively, but it is evaluated as a conventional surgery. The first rectal resection intended for radical treatment is generally known to have been performed by WE Miles in $1907^{[1]}$.

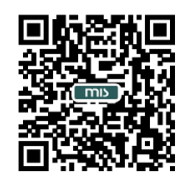


At the same time, as distal resections including for rectal cancer, the Hartmann operation was performed by Henri A. Hartmann in 1921 and the anterior resection by Dixon in 1948. In 1967, the "No-touch isolation technique" was introduced by Rubert Turnbull Jr., and in the same year the circular stapler was first introduced by the Russian pediatric surgeon Mark Mitchell Ravitch, making it widely used for convenient and easy anastomosis. In 1982, Bill Heald introduced the concept of total mesorectal excision (TME), which led to a dramatic improvement in the local recurrence rate. Since then, the Holy plane has been the gold standard for rectal cancer surgery ${ }^{[2]}$.

In 1998, Maas et al. ${ }^{[3]}$ reported that autonomic nerves sparing could reduce the rate of postoperative urinary and sexual dysfunction.

Recently, with the development of minimally invasive surgery such as laparoscopic surgery and robotic surgery, favorable results of several studies (COST, CLASSIC, COLOR II, and COREAN trials) have been published one after another ${ }^{[4,5]}$.

\section{HISTORY OF TRANSANAL APPROACH}

As mentioned above, transanal resection of rectal cancer was first performed by Jacques Lisfranc in 1826 and was accompanied by a transanal or transperineal approach as a form of combined surgery. In 1983, transanal endoscopic microsurgery (TEM) was introduced by Gerhard Buess. The system is inserted into the anus using a metallic cylinder with a beveled end (proctoscope) of 40-mm diameter and can be operated using laparoscopic instruments under a three-dimensional view after setting. In 2010, Atallah et al. ${ }^{[6]}$ introduced TransAnal Minimally Invasive Surgery (TAMIS), a hybrid of TEM and single port laparoscopic surgery.

This type of operation is advantageous because it enters the surgical field of view at both the end lumen and the side wall of the intestinal tract, compared to the TEM, which has only the cylinder end area as the operation field and a relatively short port length. In addition, it is larger and can provide a free range of motion $(\mathrm{ROM})^{[7]}$.

Furthermore, experimental transanal total mesorectal excision (TaTME) surgeries were performed, mostly on swine or cadaver. In 2010, the first laparoscopic transanal TME was reported by Sylla et al. ${ }^{[8]}$ after the first clinical application in 2009. They used a two-team approach, transabdominal and transanal. While the transabdominal team performed multiport laparoscopic colonic dissection and vessel ligation, the transanal team met in the middle with TaTME through the anus ${ }^{[8]}$.

In 2013, Leroy et al. ${ }^{[9]}$ and Zhang et al. ${ }^{[10]}$ reported surgery for rectal lesions using natural orifice transluminal endoscopic surgery (NOTES) by transanal TME without transabdominal assistance. Leroy et al. ${ }^{[9]}$ used TEO system and Zhang et al. ${ }^{[10]}$ used soft single port.

In 2014, Chouillard et al. ${ }^{[11]}$ reported small series of transanal "down-to-up" TME without any form of abdominal assistance, namely the pure NOTES for rectal cancer. According to their experience, among the total trial of 16 patients, operations on 10 patients could be finished by pure NOTES.

Since then, TaTME approaches to rectal cancer have been developed in various ways around the world. In addition, some trials have already been made to combine robotic surgery. Recently, the pure NOTES method for rectal cancer has been further developed. We performed transanal total proctocolectomy with IPAA for triple colorectal cancer without abdominal assistance in 2017. This may be the end point or final goal of minimally invasive surgery ${ }^{[12]}$. 


\section{CONCERNS ON TRANSANAL APPROACH}

\section{TAMIS}

\section{Natural orifice specimen extraction}

When the operation is performed by the transanal approach, the specimen should be discharged through the anus. In addition, transanal or transvaginal extraction is possible after conventional laparoscopy using the transabdominal approach. In the case of benign lesion or specimen by local excision, it is easy to remove, but, when the lesion itself is large or the specimen is bulky by TaTME, transanal extraction is relatively impossible due to the condition of anal sphincter (specimen-sphincter mismatch). In this case, it is necessary to switch to transabdominal extraction. In some cases, it can be safely removed by gentle and slow dilation; otherwise, excessive removal can cause sphincter injury and eventually lead to dysfunction $^{[13,14]}$.

While transanal extraction has the advantage that it can be applied irrespective of gender, transvaginal extraction is only possible in women and in some cases. It is also known that the rate of protective ileostomy is higher because of the relatively difficult incisions and the associated complications during the removal of the extract ${ }^{[15]}$.

However, there are advantages in that relatively large extracts, for example those after RHC, can be taken out without large incisions and sphincter injury can be avoided ${ }^{[16]}$.

\section{Technical notes}

Initially, a combination of surgical glove and wound protector was rolled down to create a homemade type port. Later, readymade ports were introduced, and the SILS port, OCTO port, and mini port were used, sequentially. Recently, however, a combination of Globe port and PPH's circular anal dilator has been used. This combination is easier and cheaper to install than the TEM system, and it is also superior in terms of view, as it can see the side of the view that cannot be seen in the TEM system at the same time. It is also advantageous for technical manipulation, as Atallah explained, with much more freedom and wider scope of application (ROM).

Instead of an Airseal system, a homemade reservoir system using surgical gloves can be manufactured easily and quickly. In this case, it is useful to secure a stable operative field at no additional cost [Figure 1].

TAMIS is a single port laparoscopic surgery format. Therefore, an inevitable jam is caused because the narrow space must be shared with the camera assistant. This is inconvenient for both the operator and the assistant. The answer to this inconvenience is solo surgery. The camera holder can be secured to the bed rail and the procedure can be performed without an assistant according to operator's own control and fixed focus [Figure 2].

The level of lesion available with this type of surgery (TAMIS) is commonly considered as mid or low rectal lesion. In practice, however, techniques such as access and excision are possible for lesions that exist at higher heights. The peritoneal reflection is usually regarded as a height equivalent to the second Houston valve level with some variation. Proximal lesions at higher heights are more likely to be perforated when resected into the whole layer, and infiltration of air into the abdominal cavity may cause difficulty in securing a stable field of vision and maintaining a stable surgical field. There is also a possibility of contamination.

Given the structure of the peritoneal reflex, the probability of perforation is relatively high, especially in lesions present in the anterior aspect. 


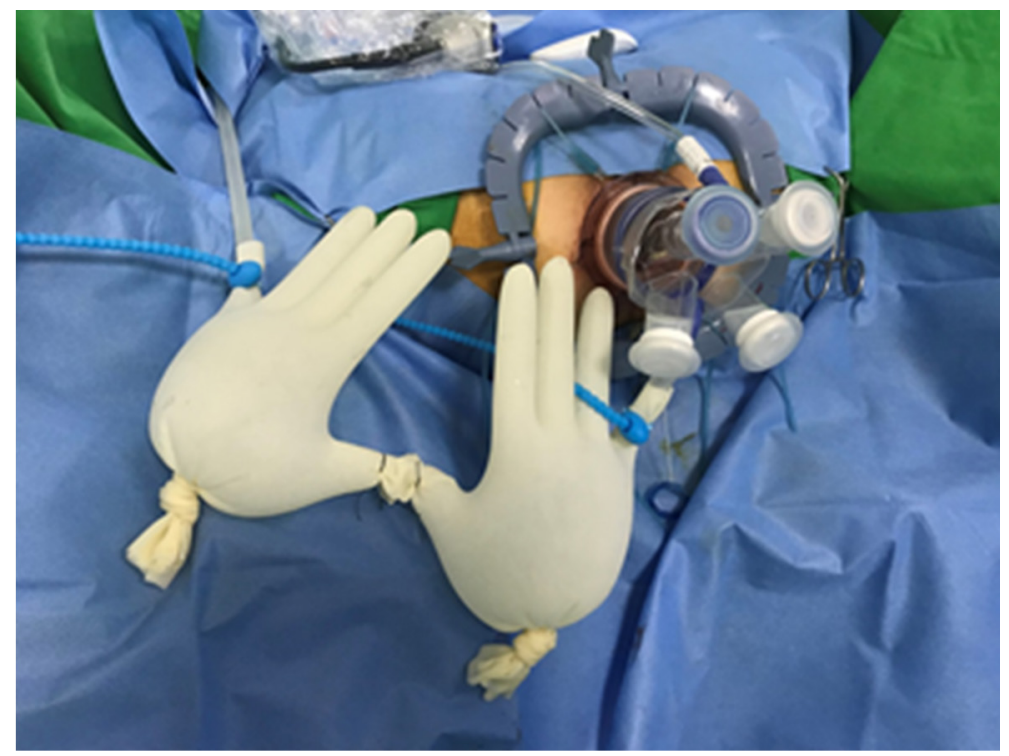

Figure 1. Surgical gloves as a reservoir. Powder free surgical glove can be used as a reservoir instead of Airseal system. Using a pair of gloves is good enough and provides stable pneumo-rectum/pelvis during transanal procedures

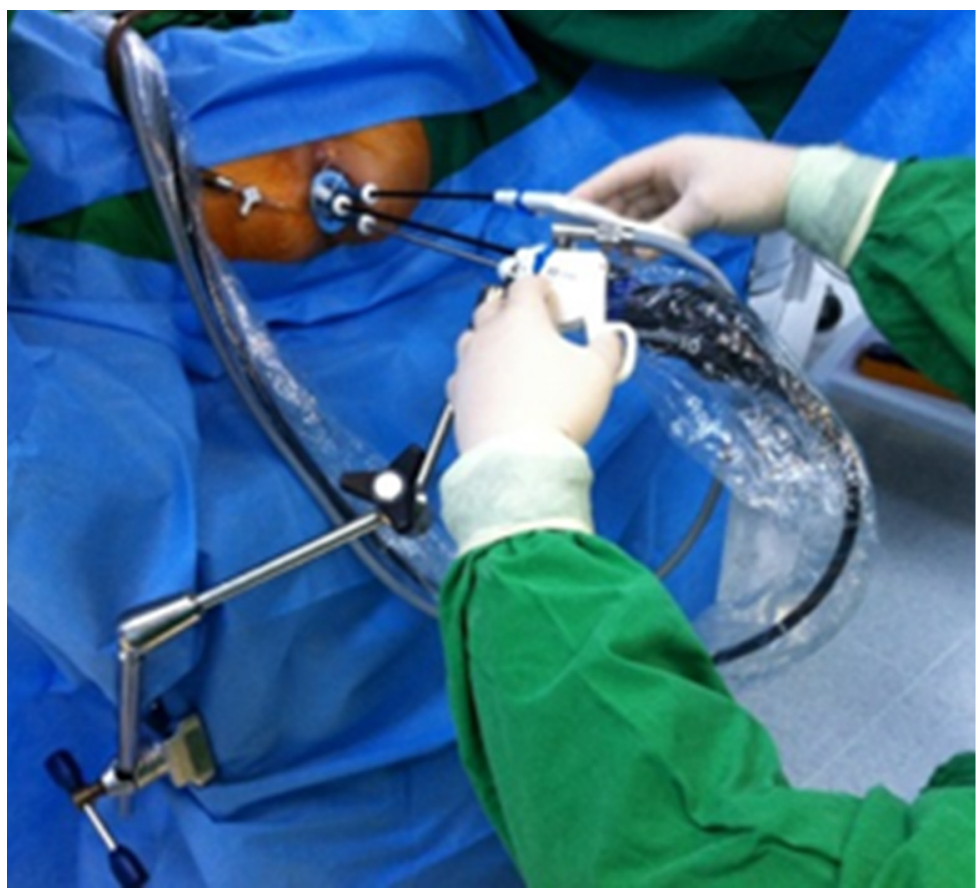

Figure 2. Solo surgery using a camera holder. Inevitable jam during single port laparoscopic surgery can be solved by applying the solo surgery. With the help of a camera holder fixed to rail of bed, the operator can use the space freely for stable procedures

Once perforation occurs, suture is possible even under TAMIS conditions, so it can be repaired by transanal manipulation. However, if the injury is large or there is already contamination of the abdominal cavity by the intestinal contents, repair and lavage may be necessary by the transabdominal approach. In this case, a single port laparoscopic procedure is usually preferred. Once the transabdominal approach is added, the length of stay is usually longer. 
Patient position is also important in TAMIS surgery. In general, it is true that positioning the lesion at the bottom is convenient and advantageous for surgery. However, it is not necessary to vary the position of the patient to place the lesion at 6 o'clock as in TEM conditions, or to adjust the system to center the field of vision during surgery.

Wherever the lesion is located under the lithotomy position, it is relatively easy to access, but some authors still mention that the prone position is helpful for anterior proximal lesions ${ }^{[17]}$.

Albert et al ${ }^{[18]}$ first suggested that the lesions be placed below when introducing the TAMIS, but a recent publication stated that lithotomy position could resolve all lesions.

Even in malignant lesions, local recurrence rate is low in lesions within $\mathrm{T} 1$, but local recurrence rate is significantly increased in $\mathrm{T} 2$ lesions.

\section{NCCN guidelines for rectal cancer: local excision}

Current guidelines for local excision of rectal cancer, e.g., the criteria for including the use of TAMIS, are based on long-term survival and outcome data. National guidelines recommend transanal local excision of only those T1No rectal cancers that meet the following criteria: $<30 \%$ circumference of bowel, $<3 \mathrm{~cm}$ in size, $>3-\mathrm{mm}$ margins, mobile, nonfixed, within $8 \mathrm{~cm}$ of anal verge, endoscopically removed polyp with cancer or indeterminate pathology, no negative pathologic features such as lymphovascular or perineural invasion, no evidence of lymphadenopathy on pretreatment imaging, and tumors that are well to moderately differentiated. Furthermore, they recommend that local excision of more proximal lesions would be technically feasible using transanal microscopic surgery or TAMIS ${ }^{[19]}$.

\section{FUTURE AND THE FINAL GOAL OF TRANSANAL MINIMALLY INVASIVE SURGERY NOTES for rectal cancer}

When the pelvic delamination is complete through TaTME, this space can serve as a common path for access into the abdominal cavity. Under these conditions, various organs and intestines can be accessed from the abdominal cavity and surgical procedures can be performed. This is NOTES if the resulting extract is removed through the anus ${ }^{[20]}$.

This procedure consists of three steps: anal, intraperitoneal, and second anal stage. In the first stage, TaTME takes place. In the second stage, vascular and mesenteric dissection is performed simultaneously with colonic mobilization. If necessary, splenic flexure mobilization is also performed. Finally, in the third stage, specimen pull through, transection, and anastomosis occur [Figure 3].

However, to approach the intraperitoneal through the transanal approach, there are some challenges that must be considered as well as some difficulties to overcome in the technical aspect. First, the condition of the anal sphincter should be compared with the characteristics of the lesion. The anus should be sufficiently intact and allow for safe passage of the extract, including the lesion. The second is the prominence of pelvic promontory. Usually, the transanal approach to inferior mesenteric artery (IMA) and inferior mesenteric vein (IMV) is possible without major difficulties. However, in some older patients, there are severe bends and protrusions of the pelvic promontory. This acts as a major obstruction to accessing the abdominal cavity and, in severe cases (in cases where it is impossible to attempt a straightening of the spine by changing the patient's position), a transabdominal approach should be added. Third, long shafted devices should be prepared for possible splenic flexure mobilization, as well as smooth manipulation of IMA and IMV. Commercially available laparoscopic instruments can reach up to $46 \mathrm{~cm}$. If further distances are predicted from the patient's radiology data, surgery by NOTES should be considered difficult [Figure 4]. 

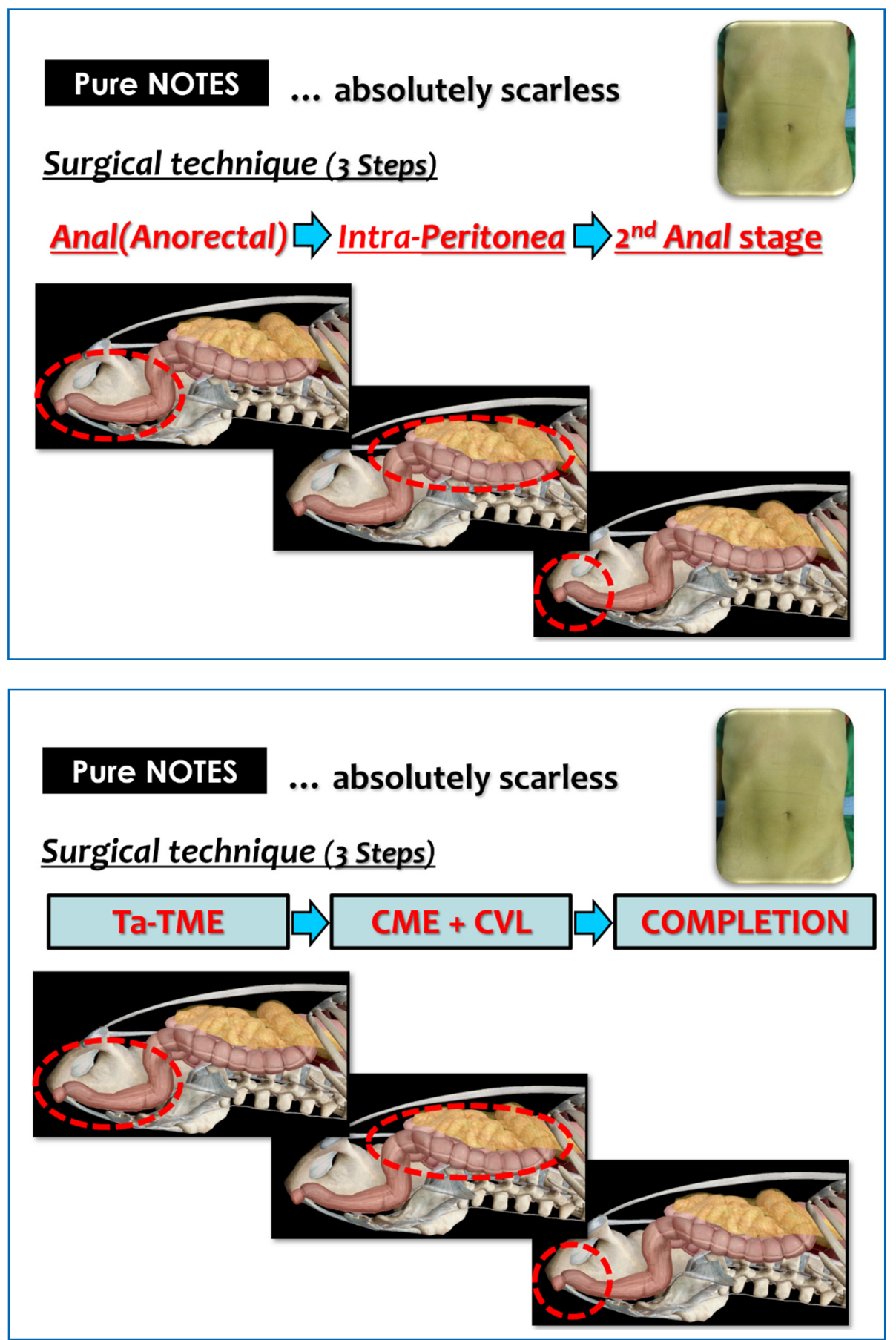

Figure 3. Three steps of pure NOTES. Pure NOTES consist of three steps: anal (anorectal), intracorporeal, and second anal step. According to each step, TaTME, CME $+C V L$, and anal completion are performed, respectively. Anal completion can be done by finishing of pull through, transection of specimen, and anastomosis. NOTES: natural orifice transluminal endoscopic surgery; TaTME: transanal total mesorectal excision; CME: complete mesocolic excision; CVL: central vascular ligation

In addition, NOTES-style surgery may be difficult under conditions such as redundancy of the colon length, anatomical variation, and surgical history including urological gynecology, and it is difficult to predict the future, such as severe natural adhesion of the colon to the left low quadrant (LLQ) area or excessive extension. Care should be taken to prevent or reduce the possibility of soiling or spillage. The author's method is to use a Lap-bag (Endo-catch bag). With sufficient TME in the pelvic cavity, entry into the abdominal cavity is necessary for further dissection and progression. At this time, an incision is made in the anterior peritoneal reflexion through the abdominal cavity and pushed into the abdominal cavity 


\section{Pure NOTES}

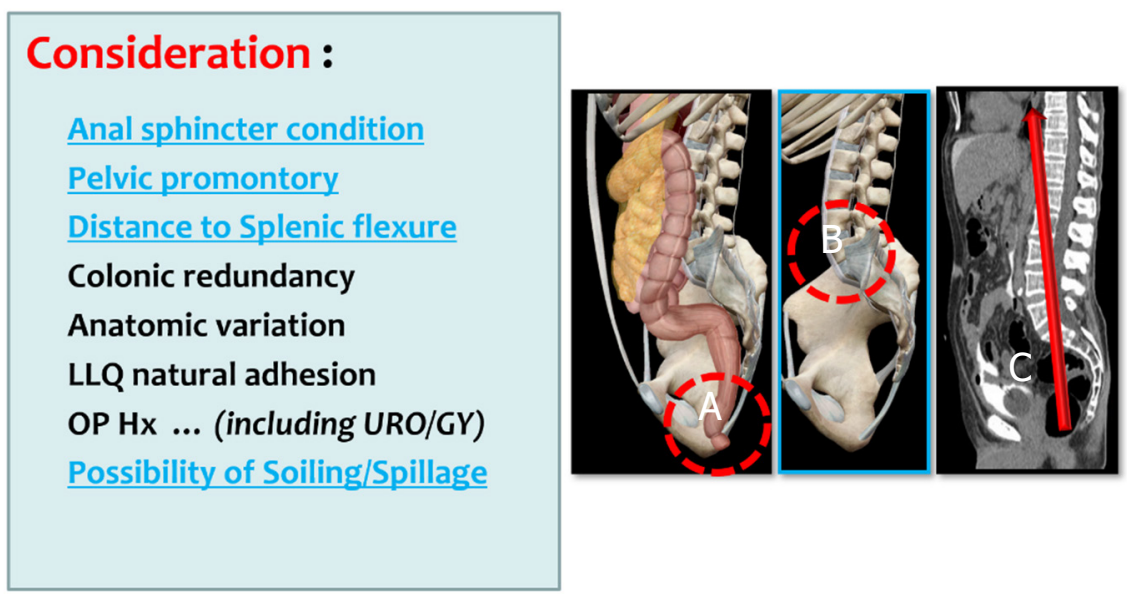

Figure 4. Considerations in pure NOTES. Preoperative considerations are anal sphincter condition (A), pelvic promontory (B), distance to splenic flexure (C), operative history, etc. NOTES: natural orifice transluminal endoscopic surgery; LLQ: left low quadrant
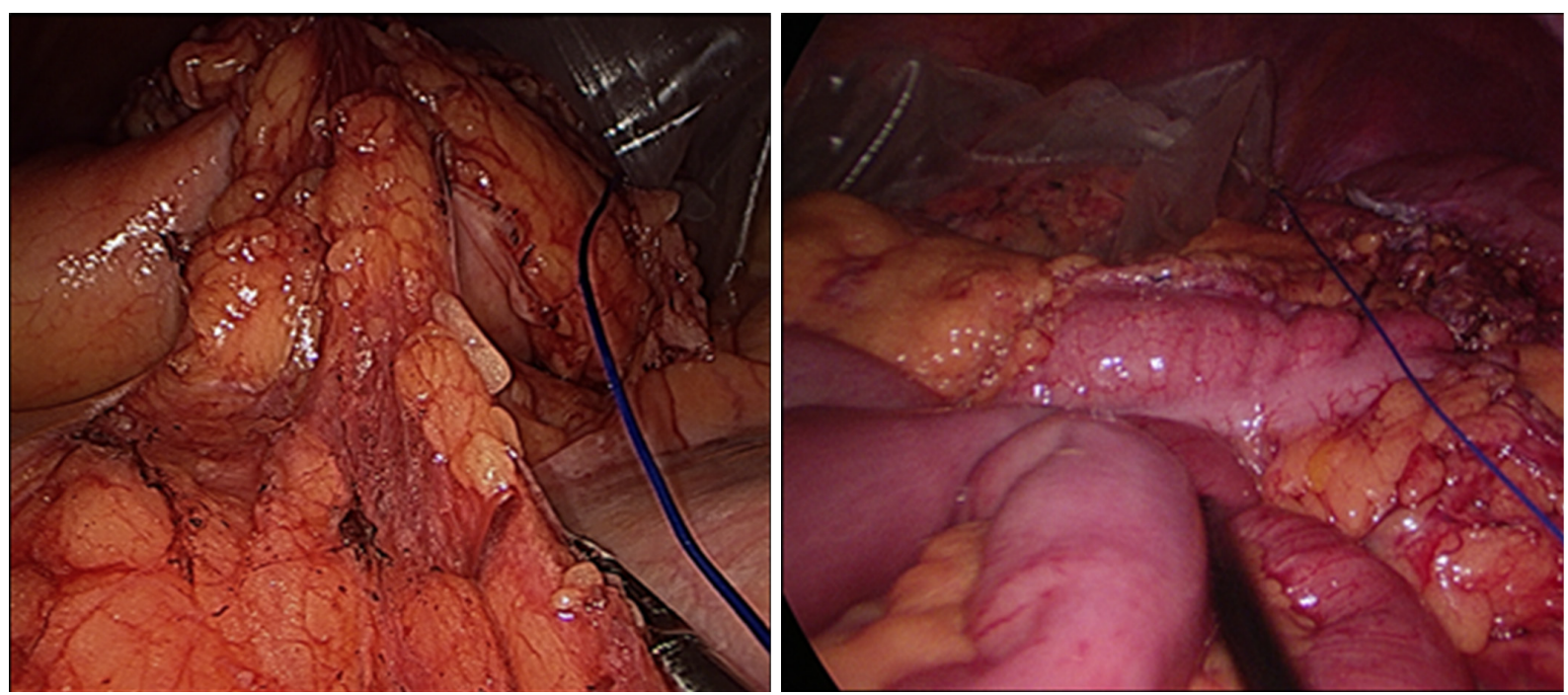

Figure 5. Lap-bag application. Especially Lap-bag should be considered for prevention from soiling and spillage during intraperitoneal step

with a rectal stump in the Lap-bag. This prevents soiling or spillage and at the same time improves the field of view because the rectal stump, including the lesion, is out of the center of the field of view [Figure 5].

In general, Hemo-loc or clip is usually used for ligation of IMA or IMV in laparoscopic surgery. However, when implementing transanal NOTES, a series of operations such as loading, entering, and firing must be performed several times in succession, and the moving range is relatively long. In this process, there is the possibility of damaging surrounding organs or tissues by blindly reciprocating a fairly long section. In contrast, when an energy device with a good sealing effect is used, the procedures can be carried out continuously by using "Overlapping sealing technique" in the field without repeating the work carried out during use of Hemo-loc or clips. Sealing power and security are also firm and safe enough. This can be said 

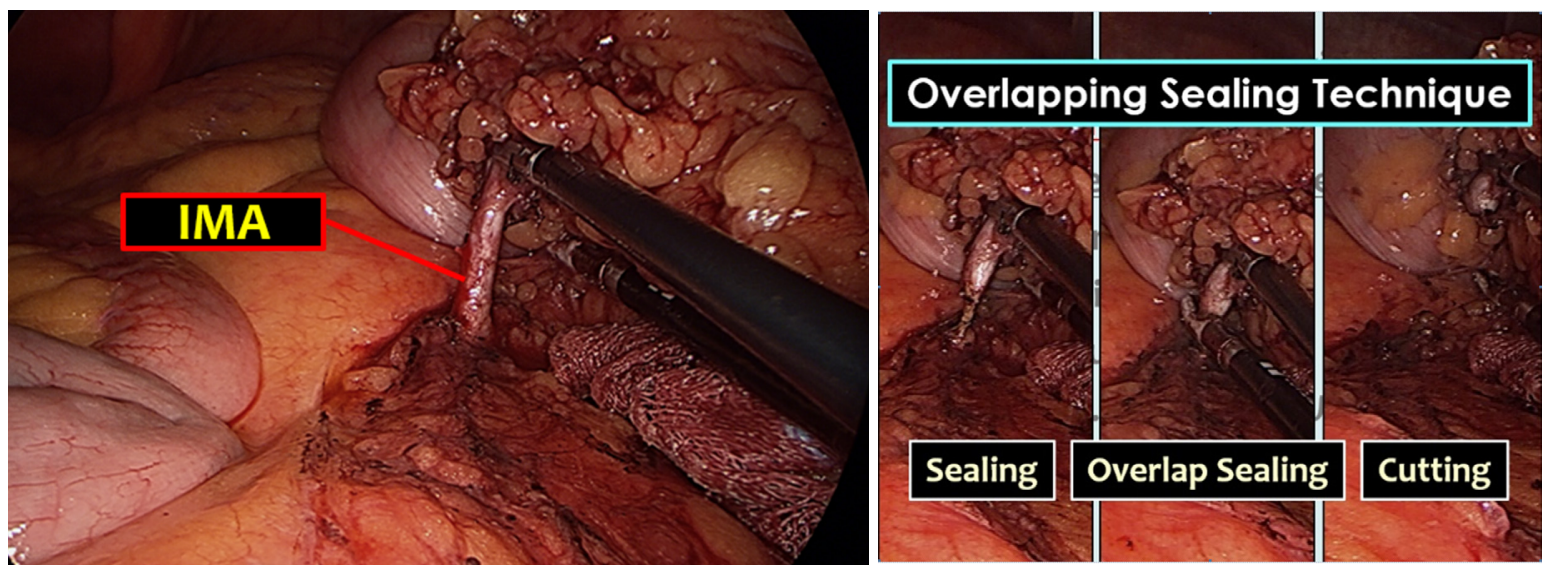

Figure 6. Overlapping sealing technique. Using of energy device instead of Hemo-loc or clip is beneficial to save time and reduce possible complications related with Hemo-loc or clip. "Overlapping sealing technique" may increase security. IMA: inferior mesenteric artery
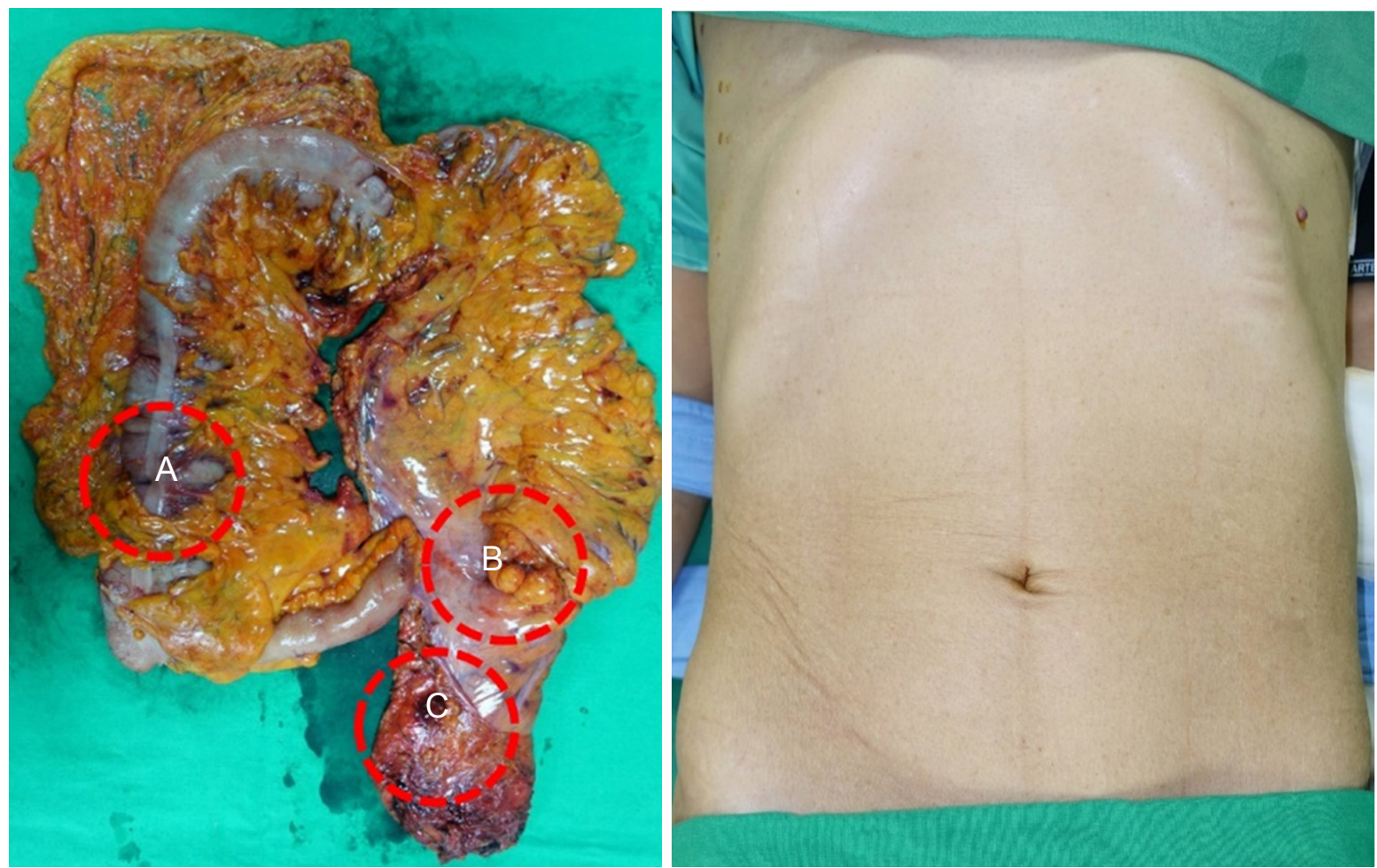

Figure 7. Specimen and post-OP abdominal view. Even though a large-scale operation produces a bulky specimen, there is absolutely no visible operative incision or wound. Circles indicate Ascending colon cancer (A), Sigmoid colon ca (B), and Rectal cancer lesions respectively (C)

to be a reasonable advantage as it not only saves time but also reduces complications that can occur during entry and exit [Figure 6].

\section{Reported case: transanal total proctocolectomy with IPAA}

In February 2017, we performed transanal total proctocolectomy with IPAA without abdominal assistance in patients with triple colorectal cancers (ascending, sigmoid colon, and rectum) using pure NOTES $^{[12]}$ [Figure 7].

\section{Case summary}

A 70-year-old male patient without specific medical history was diagnosed with synchronous triple colorectal cancers (ascending colon, rectosigmoid colon, and rectum). We performed transanal total 

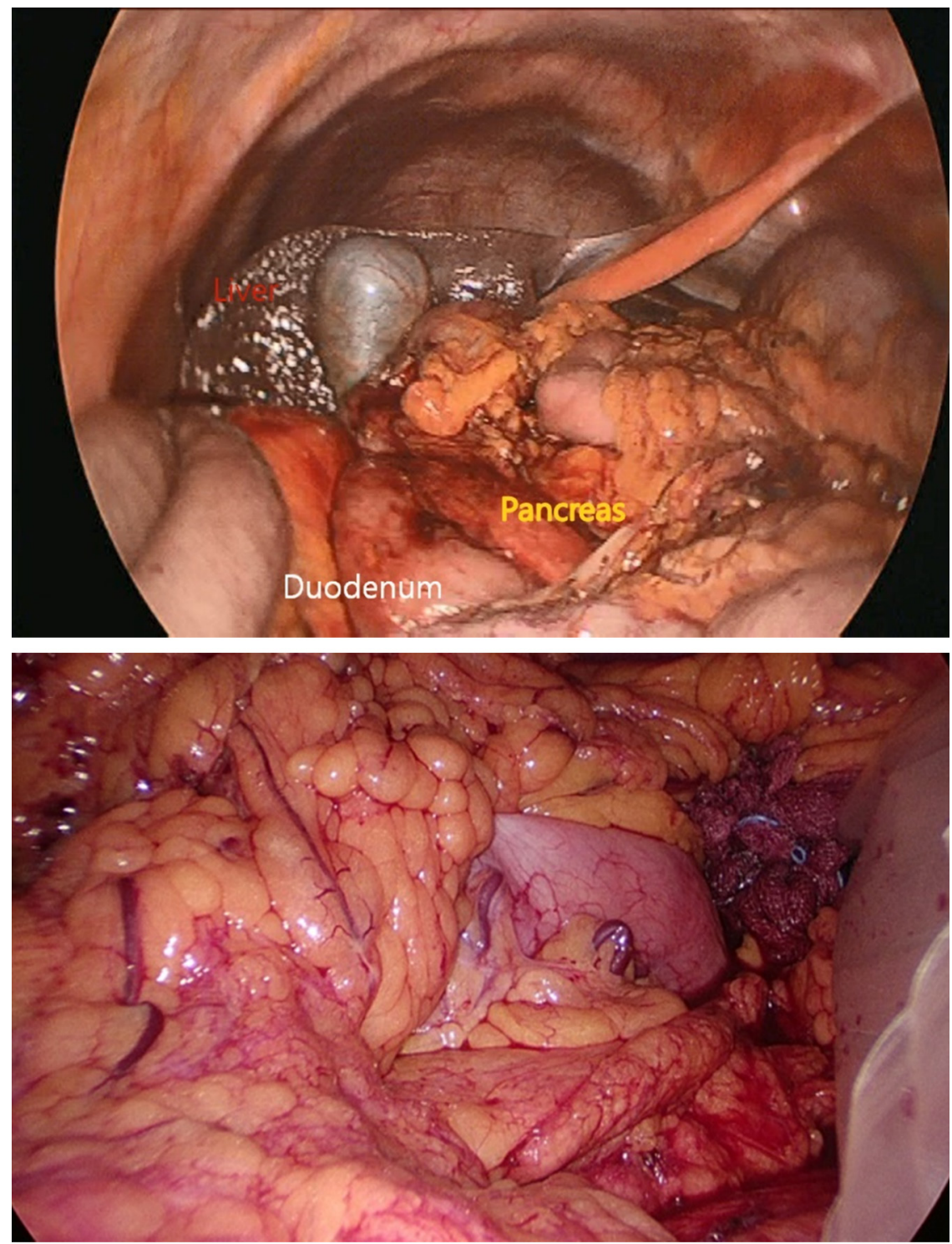

Figure 8. Transanal hepatic and splenic flexure mobilization. These are somewhat unique afterviews of transanal hepatic and splenic flexure mobilization

proctocolectomy with ileal pouch-anal anastomosis. On preoperative MRI, there was no pelvic lateral lymph node, thus we did not need to perform chemoradiation therapy. After transanal dissection of the mesorectum, rectum was flipped into the intraperitoneal space for further dissection. In our setting, we used conventional laparoscopic instruments for most procedures and long-shafted instruments helped during mobilization of the splenic and hepatic flexures [Figure 8].

The entire specimen was extracted transanally. The ileal pouch was constructed intracorporeally using two cartridges of linear staplers and ileal pouch-anal anastomosis was performed using a $25-\mathrm{mm}$ circular stapler. We did not create a defunctioning stoma. Total operating time was $328 \mathrm{~min}$ and blood loss was $<50 \mathrm{~mL}$. These were based on anesthesiologist records. We harvested 61 lymph nodes, and one regional lymph node metastasis was found. The patient experienced temporary paralytic ileus, was discharged on Postoperative Day 10, and had no major complications. The patient received antidiarrheal drug but had no incontinence. The patient refused adjuvant chemotherapy. During the 32-month follow-up period, there were no recurrences or metastases during five colonoscopies and CT scans. This operation was performed 
in February 2017 and this pure NOTES transanal total colectomy has been the first case and is a unique case thus far.

\section{NOTES for intraperitoneal organs}

NOTES methods for accessing the abdominal cavity may include transanal and transvaginal routes, and, especially in patients with stoma, the stoma may be recognized as one of the natural openings. For the transanal approach, matching between the specimen size including the mass and the accommodation of the sphincter is important, and, for the transvaginal approach, virginity or parity can be an important consideration. The transvaginal approach is advantageous for the extraction of quite large specimens, especially specimens made with en-bloc resection. It also has an advantageous approach in terms of angle with the abdominal cavity. Postoperative sutures may also be easier than intestinal anastomosis performed by transanal access surgery, and relatively less burden on leakage. In patients with stoma, the stoma can be recognized as one of the natural openings in a broad sense. The abdominal cavity can be accessed by taking down the stoma or partial splitting the margin of the stoma without inflicting any other wound. This is done by performing a single laparoscopy and finishing again with stoma to maintain the same condition as before.

In other surgical areas, various NOTES-type surgeries are newly introduced and performed. Surgery for various organs and intestines in the abdominal cavity is ultimately developing as absolutely scarless surgery. Some of them are already making significant progress, and, in cancer surgery, homework remains a matter of approach and adherence to oncological laws as in any surgery.

\section{DECLARATIONS}

\section{Authors' contributions}

Performed surgeries and contributed to conception and design of the study: Lee SC

Performed data acquisition and analysis, as well as provided technical support: Choi BJ

Wrote the thesis: Jeong WJ

\section{Availability of data and materials}

Not applicable.

\section{Financial support and sponsorship}

None.

\section{Conflicts of interest}

All authors declared that there are no conflicts of interest.

\section{Ethical approval and consent to participate}

Not applicable.

\section{Consent for publication}

Not applicable.

\section{Copyright}

(c) The Author(s) 2019.

\section{REFERENCES}

1. Lange MM, Rutten HJ, van de Velde CJ. One hundred years of curative surgery for rectal cancer: 1908-2008. Eur J Surg Oncol 2009;35:456-63

2. Lirici MM, Hüscher CG. Techniques and technology evolution of rectal cancer surgery: a history of more than a hundred years. Minim 
Invasive Ther Allied Technol 2016;25:226-33.

3. Maas CP, Moriya Y, Steup WH, Kiebert GM, Kranenbarg WM, et al. Radical and nerve-preserving surgery for rectal cancer in The Netherlands: a prospective study on morbidity and functional outcome. Br J Surg 1998;85:92-7.

4. Van der Pas MH, Haglind E, Cuesta MA, Fürst A, Lacy AM, et al. Laparoscopic versus open surgery for rectal cancer (COLOR II): short-term outcomes of a randomised, phase 3 trial. Lancet Oncol 2013;14:210-8.

5. Jeong SY, Park JW, Nam BH, Kim S, Kang SB, et al. Open versus laparoscopic surgery for mid-rectal or low-rectal cancer after neoadjuvant chemoradiotherapy (COREAN trial): survival outcomes of an open-label, non-inferiority, randomised controlled trial. Lancet Oncol 2014;15:767-74.

6. Atallah S, Albert M, Larach S. Transanal minimally invasive surgery: a giant leap forward. Surg Endosc 2010;24:2200-5.

7. Atallah S, Albert M, Monson JR. Critical concepts and important anatomic landmarks encountered during transanal total mesorectal excision (taTME): toward the mastery of a new operation for rectal cancer surgery. Tech Coloproctol 2016;20:483-94.

8. Sylla P, Rattner DW, Delgado S, Lacy AM. NOTES transanal rectal cancer resection using transanal endoscopic microsurgery and laparoscopic assistance. Surg Endosc 2010;24:1205-10.

9. Leroy J, Barry BD, Melani A, Mutter D, Marescaux J. No-scar transanal total mesorectal excision: the last step to pure NOTES for colorectal surgery. JAMA Surg 2013;148:226-30; discussion 231.

10. Zhang H, Zhang YS, Jin XW, Li MZ, Fan JS, et al. Transanal single-port laparoscopic total mesorectal excision in the treatment of rectal cancer. Tech Coloproctol 2013;17:117-23.

11. Chouillard E, Chahine E, Khoury G, Vinson-Bonnet B, Gumbs A, et al. NOTES total mesorectal excision (TME) for patients with rectal neoplasia: a preliminary experience. Surg Endosc 2014;28:3150-7.

12. Jeong WJ, Choi BJ, Lee SC. Transanal total proctocolectomy with ileal pouch-anal anastomosis for synchronous triple colorectal cancer. J Laparoendosc Adv Surg Tech Videoscopy 2019; Epub ahead of print. Available from: http://doi.org/10.1089/ vor.2018.0566 [Last accessed on 9 Dec 2019]

13. Kim SJ, Choi BJ, Lee SC. A novel single-port laparoscopic operation for colorectal cancer with transanal specimen extraction: a comparative study. BMC Surg 2015;15:10.

14. Choi BJ, Lee SC, Kang WK. Single-port laparoscopic total mesorectal excision with transanal resection (transabdominal transanal resection) for low rectal cancer: initial experience with 22 cases. Int J Surg 2013;11:858-63.

15. Franklin ME Jr, Liang S, Russek K. Natural orifice specimen extraction in laparoscopic colorectal surgery: transanal and transvaginal approaches. Tech Coloproctol 2013;17 (Suppl 1):S63-7.

16. Park JS, Choi GS, Kim HJ, Park SY, Jun SH. Natural orifice specimen extraction versus conventional laparoscopically assisted right hemicolectomy. Br J Surg 2011;98:710-5.

17. Keller DS, Haas EM. Transanal minimally invasive surgery: state of the art. J Gastrointest Surg 2016;20:463-9.

18. Albert MR, Atallah SB, deBeche-Adams TC, Izfar S, Larach SW. Transanal minimally invasive surgery (TAMIS) for local excision of benign neoplasms and early-stage rectal cancer: efficacy and outcomes in the first 50 patients. Dis Colon Rectum 2013;56:301-7.

19. NCCN guidelines version 3.2019 rectal cancer. NCCN Clinical Practice Guidelines in Oncology. Available from: https://www.ncen.org/ professionals/physician_gls/default.aspx [Last accessed on 9 Dec 2019]

20. Jeong WJ, Choi BJ, Lee SC. Pure natural orifice transluminal endoscopic surgery for rectal cancer: Ta-TME and CME without abdominal assistance. J Asian J Surg 2019;42:450-7. 